\title{
Handling Human-Centered Systems Uncertainty Using Fuzzy Logics - A Review
}

\author{
Isabel L. Nunes*
}

\author{
Universidade Nova de Lisboa/ Faculdade de Ciências e Tecnologia, Departamento de Eng. Mecânica e Industrial, \\ Campus de Caparica, 2829-516 Caparica, Portugal
}

\begin{abstract}
The analysis of human-centered systems (HCS) is complex, involving vagueness, uncertainty and ill-defined data. The subjective nature of human classifications renders classical logics approaches almost useless to deal with HCS. Fuzzy Logics (FL) provides a mathematical framework for the systematic treatment of vagueness and imprecision; facilitates the elicitation and encoding of uncertainty-related knowledge; and a flexible representation mechanism for dealing with vague data. The broad variety of fuzzy operators covers the continuum from union operators to intersection operators, including average operators. This paper presents several application examples of FL in HCS analysis, namely related with Ergonomics and Safety Analysis.
\end{abstract}

Keywords: Human-centered Systems, fuzzy logics, ergonomics, safety.

\section{INTRODUCTION}

Human-Centered Systems (HCS) is understood as the system Human-Machine-Environment, i.e., the people at work and their relations with the surrounding environment, equipment and fellow workers. The analysis of such systems can be performed aiming different goals - like safety, health, human reliability, stress or usability-, and may be addressed from different perspectives - like the physical, physiological, organizational, cognitive, or socio-psychological.

A HCS intervention can be performed at a design or redesign phase of a work system, and is focused on identifying incompatibilities between work requirements and hazards, and human capabilities. The objective is eliminating the incompatibilities or, at least, reducing them, so that working systems become safer, healthier, more productive, comfortable, and satisfying [1].

Is a basic principle in the law of many countries that employers have to take the necessary measures for the safety and health protection of workers. For instance, within the European Community, the legal framework was settled by the Council Directive of 12 June 1989 on the introduction of measures to encourage improvements in the safety and health of workers at work (89/391/EEC), and then adopted by Member States' national laws. For this purpose employers must analyze HCS regarding safety and health at work, and decide on preventive measures to take and, if necessary, protective equipment to use.

The knowledge required to analyze HCS is multidisciplinary and the data used to evaluate them is often characterized by being complex, imprecise, uncertain and vague. The problem complexity and data vagueness (for

*Address correspondence to this author at the Universidade Nova de Lisboa/ Faculdade de Ciências e Tecnologia, Departamento de Eng. Mecânica e Industrial, Campus de Caparica, 2829-516 Caparica, Portugal; Tel: +351 21 29485 42; Fax: +351 2129485 46; E-mail: imn@fct.unl.pt instance, the ill-definition resulting from the subjective nature of human classifications) renders classical logics approaches almost useless to deal with HCS.

Fuzzy Logics (FL) provides an appropriate logicalmathematical framework to handle problems with such characteristics, since [2]:

- deals with uncertainty and imprecision of reasoning processes;

- allows the modeling of the heuristic knowledge (that cannot be described by traditional mathematical equations); and

- allows the computation of linguistic information.

The aim of this paper is to divulge examples of the application of FL in HCS highlighting the advantages that arise from the use of the fuzzy methodology.

This paper presents a survey on the publications issued on the last 12 years regarding the use of fuzzy logic in human-centered systems. The sources were Portuguese and English language edited books and papers retrieved from searches in databases such as B-on, ISI Web of knowledge and INSPEC, using expressions such as: "FL and Ergonomics", "FL and Safety" or "FL and occupational stress".

The following sections of paper will provide a brief overview about Fuzzy Logics, present several examples of fuzzy systems applied in the assessment of HCS-related issues, namely, comfort, human reliability, workload stress, health, and safety. At the end some conclusions will also be presented.

\section{FUZZY LOGICS}

Fuzzy Logic is based on the concept of fuzzy set formulated in the Fuzzy Set Theory (FST) by Lotfi Zadeh, in 1965 [3]. FST provides a mathematical framework for the systematic treatment of vagueness and imprecision. The 
subjective nature of human classification processes renders classical (Boolean/dichotomous) approaches almost useless to deal with human-centered systems. So FST facilitates the elicitation and encoding of uncertain knowledge. It provides a representation mechanism that improves the flexibility for dealing with data associated to complex concepts. The result of using FST is more robust tools that perform better for a wider variety of conditions and users. From an encoding point of view, fuzzy sets support the representation of knowledge and its uncertainty as a unique entity. The resulting representation is very flexible and it can be easily coupled with non-fuzzy forms of knowledge representation, and manipulated by a variety of evaluation methods.

A fuzzy set presents a boundary with a gradual contour, by contrast with classical sets, which present a discrete border. Formally, let $\mathrm{U}$ be the universe of discourse and $u$ a generic element of $U$, a fuzzy subset $A$, defined in $U$, is one set of the dual pairs:

$A=\left\{\left(u, \mu_{A}(u)\right) \mid u \in U\right\}$

where $\mu_{A}(u)$ is designated as membership function or membership grade $u$ in $A$. The membership function associates to each element $u$, of $U$, a real number $\mu_{A}(u)$, in the interval $[0,1]$.

There are some typical shapes for the membership functions of fuzzy sets, such as $\pi$ or trapezoidal, Bell or Gaussian, triangular, Z - shape and S - shape. Fig. (1) presents graphics that illustrate some continuous fuzzy sets defined with such shapes.

Based on these principles it is possible to evaluate the degree of membership of some observed data. Let us consider, for instance, the evaluation of the "working height" risk (as done on the AR_X system [4]). The analysis of such concept can be performed based on an objective attribute: the height to the ground of the area where a worker operates. Fig. (5a) presents an example of fuzzy set to use in such evaluation. Based on this fuzzy set is possible to observe that the "working height" risk increases with the height. There is no fall risk, i.e. the risk degree is 0 , when the height is $0 \mathrm{~m}$. This degree increases proportionally to the working height until it becomes maximal, i.e. the risk degree reaches 1 . On the depicted example working heights above $3 \mathrm{~m}$ corresponds to highly risky situations.

An important concept in FST is the one of linguistic variables [5-8]. A linguistic variable is a variable that admits as values words or sentences of a natural language, which can be represented as fuzzy sets. Linguistic variables serve as a means of approximate characterization of phenomena which are too complex or to ill-defined to be susceptible of description in precise terms. Furthermore, in the human discourse, variables are, normally, expressed by words, not by numbers. Thus, one advantage of using linguistic variables is that one can deal directly with semantic concepts of imprecise nature, with a consistent mathematical formulation.

As an example, let us consider the acceptability linguistic variable, used to collect opinions for ergonomic or risk inadequacy degree evaluation (as used in ERGO_X [9] and AR_X [4]). In the example, this linguistic variable comprehends a set of 5 linguistic terms (Good, Acceptable, Little Acceptable, Bad, Very Bad), to which correspond inadequacy degrees. Fig. (2a) presents an example of this linguistic variable, where the terms are defined as continuous fuzzy sets. However, due to considerations about the numerical efficiency related with computational systems the linguistic variable terms can also be assumed as discrete fuzzy sets. A discrete fuzzy set corresponding to the same acceptability linguistic variable is depicted in Fig. (2b).

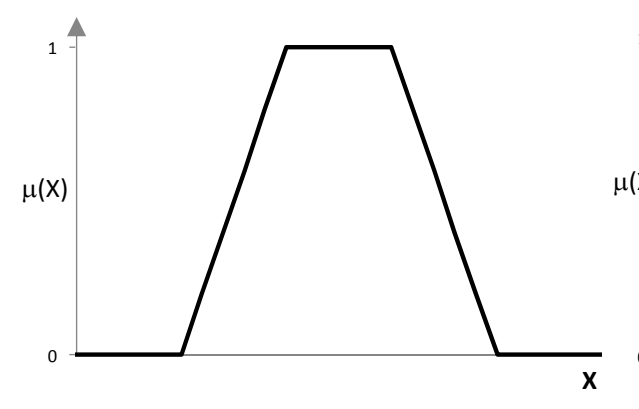

(a)

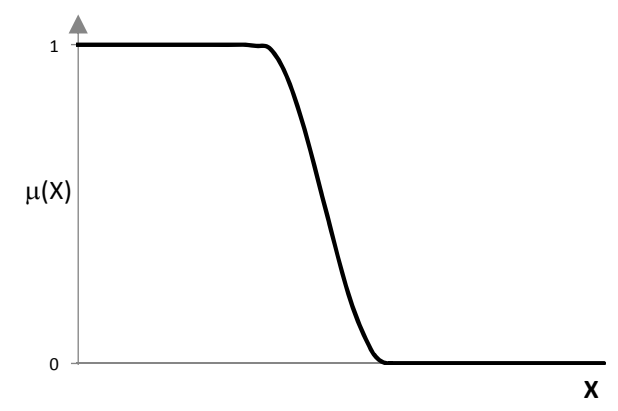

(d)

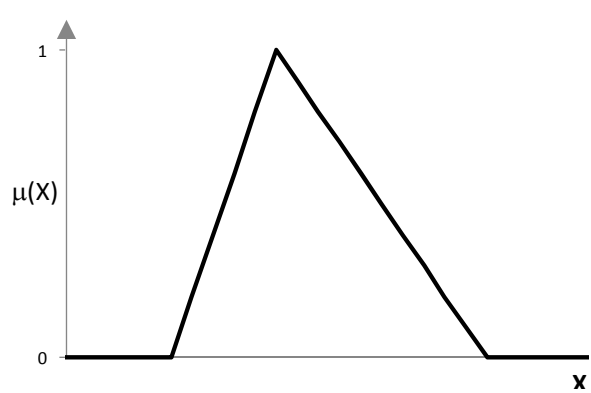

(c) (b)

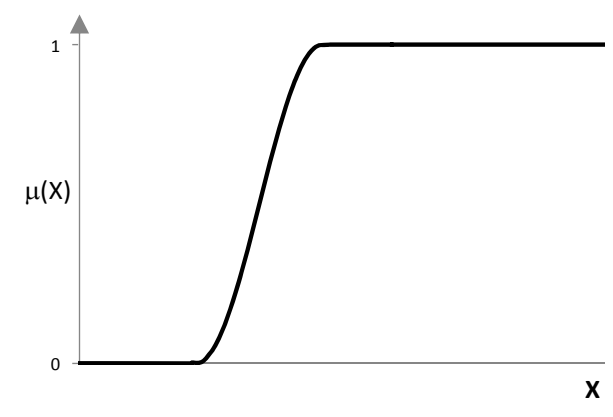

(e)

Fig. (1). Typical continuous fuzzy set membership function shapes: (a) $\pi$ or trapezoidal, (b) Bell or Gaussian, (c) triangular, (d) $Z-$ shape and (e) $\mathrm{S}$ - shape. 


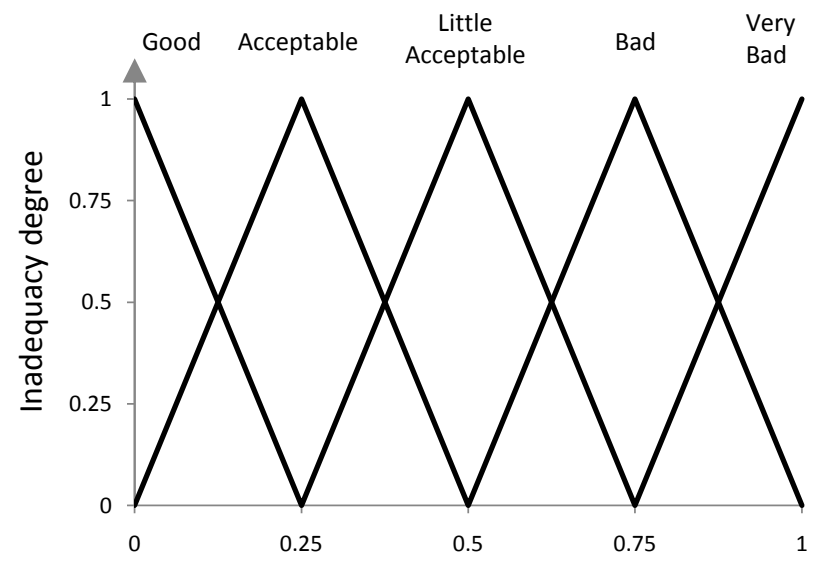

(a)

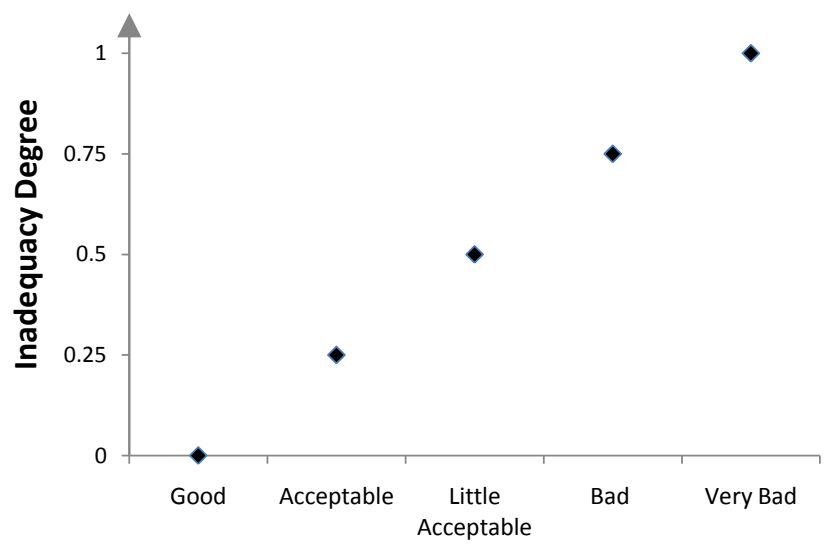

(b)

Fig. (2). Linguistic Variable acceptability (a) continuous fuzzy set terms (b) discrete fuzzy set terms.

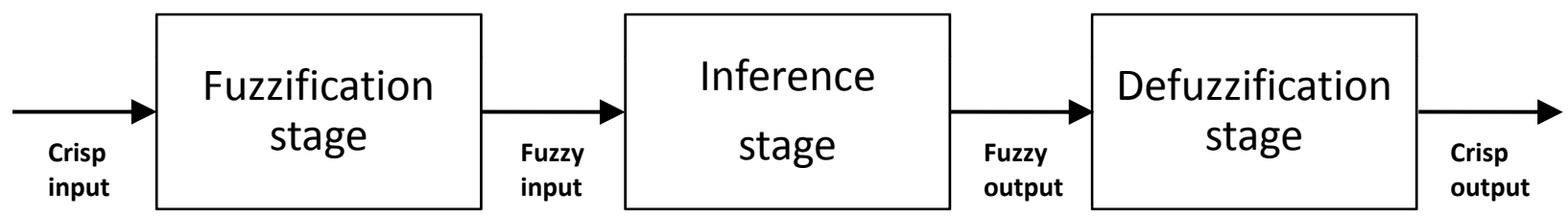

Fig. (3). Fuzzy Logic system stages.

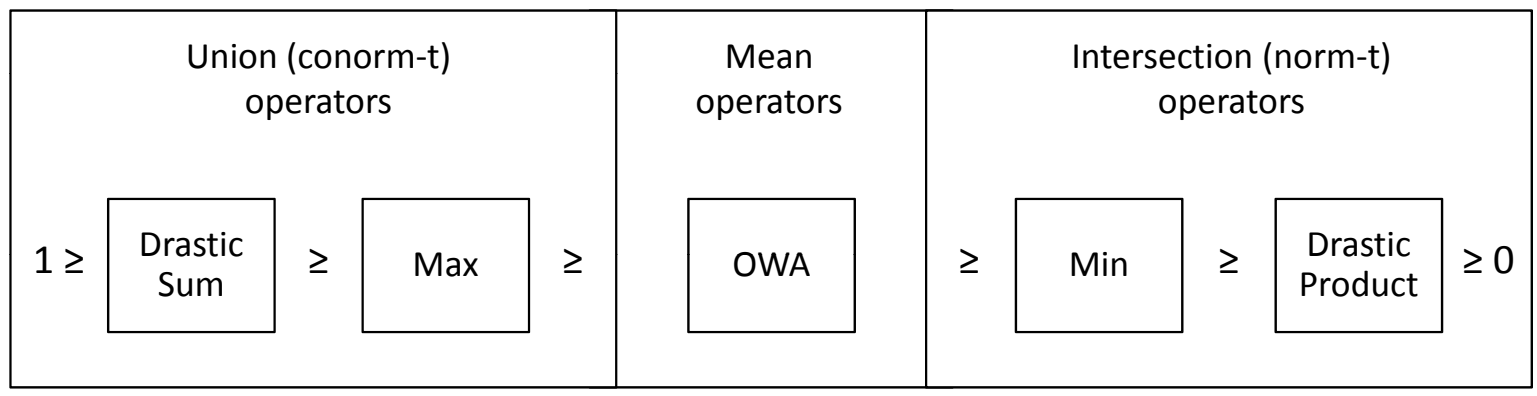

Fig. (4). Fuzzy operators continuum.

Typically the processing of a Fuzzy Logic system is performed on 3 stages (Fig. 3). The first is the fuzzification stage, where crisp input data (either numerical or linguistic) is transformed in fuzzy sets. The second is the inference stage, where fuzzy rules are applied aggregating input data by means of fuzzy logical operators and fuzzy IF-THEN rules producing fuzzy output results. The last stage, the defuzzification, corresponds to the conversion of fuzzy results into crisp results (either numerical or linguistic) to be presented to users.

Fuzzy logics admits basic arithmetic and logic operations [5] and also a set of fuzzy operators (t-norms, t-conorms and means) that provide a continuum of data aggregation behaviors which allow the simulation of several aggregation types (like, for instance, the synergy resulting of the interaction of 2 risk factors). Fig. (4) illustrates the continuum of fuzzy operators' space, referring some operators that typically define the upper and lower behaviors in each category. The fuzzy operators can be parametric or non-parametric, meaning that the results can either depend or not on the value of a parameter. Parametric operators are computationally more demanding but offer more flexibility than non-parametric operators. There is a very large variety of operators and references about them. For a thorough analysis of fuzzy operators refer, for instance, to [10].

With the wide range of fuzzy operators offered it is possible to handle most of the human-centered problems characterized by uncertainty and subjective data.

\section{EXAMPLES OF APPLICATIONS OF FUZZY LOGICS TO HUMAN CENTERED SYSTEMS}

In the following sub-sections is given a brief description of some examples of application of fuzzy logics on the fields of Occupational Safety (risk assessment for work accidents) and Ergonomics (human reliability, manual materials handling, work-related musculoskeletal disorders and layout design) identified on a literature review on reference publications issued on the last 12 years, as described in Section 1.

The examples of fuzzy systems here presented illustrate several approaches adopted in dealing with uncertainty 
reasoning as a methodology for the development of humancentered decision support systems.

\subsection{Risk Assessment for Work Accidents}

\subsubsection{Fuzzy Inherent Safety Index}

Gentile et al. proposed a fuzzy version of Heikkila's Inherent Safety Index (ISI), applied to the evaluation of chemical industry plants [11]. The Inherent Safety Index considers three factors: chemical substances, process hazard and the process equipment and tanks. Each factor is characterized by several parameters, which in the fuzzy logic model are defined as linguistic variables terms, evaluated as bell-shaped fuzzy sets. The parameters of ISI factors are presented on Table 1.

\section{Table 1. Inherent Safety Index Parameters}

\begin{tabular}{|c|c|}
\hline ISI Factors & Parameters \\
\hline \multirow{6}{*}{ Chemical Substances } & Flammability \\
\hline & Toxicity \\
\hline & Explosivity \\
\hline & Chemical interaction \\
\hline & Reactivity \\
\hline & Water \\
\hline \multirow{8}{*}{ Process Hazard } & Higher temperature \\
\hline & Higher pressure \\
\hline & Material \\
\hline & Personal Protective Equipment \\
\hline & Process Safety \\
\hline & Packing degree of the area \\
\hline & Heat of the main reaction \\
\hline & Heat of the side reactions \\
\hline \multirow{4}{*}{ Process Equipment and Tanks } & Type of process equipment \\
\hline & Type of other equipment \\
\hline & Tank volume \\
\hline & Tank's pressure \\
\hline
\end{tabular}

The evaluation of each fuzzy ISI factor is performed based on the aggregation, using an OR operator, of the results of applying a set of IF-THEN rules, based on a Mamdani model. The fuzzy output is defuzzified using a center of mass technique, resulting on a crisp output value. The evaluation of the final index is performed using a weighted sum of the individual factors outputs.

To perform the analysis of a complete chemical plant the procedure includes four steps:

1. divide the chemical plant into operating sub-processes according to the unit operations of each area;

2. for each unit identify chemical substances, operating conditions and processing equipment;

3. evaluate the ISI for each unit;
4. add the values of the indices for each area.

Compared to the Heikkila Index the Fuzzy Logic-based Index has the advantage of being sensitive to changes on the input data, since it yields continuous results, eliminating the problems presented by the traditional interval approach. In fact, the Heikkila Index inputs are discrete values classified based on range intervals, meaning that the output is the same for any input values that fall on the same interval.

\subsection{2. $A R \_X$}

Nunes presented AR_X, which is an expert system aiming to support risk analysis for work accidents. Its main objective is to identify and evaluate exposure to occupational risks and advice on measures to implement in order to control risks [4]. The methodology supports the assessment of potential factors that contribute for accident occurrence and guides the user on the adoption of corrective measures.

The AR_X will perform an Accident Risk Degree assessment based on the evaluation of protection factors and risk factors that are relevant to the situation under analysis. Table 2 presents an example for the assessment of the "fall risk" identifying the Attributes to use on the evaluation process.

Table 2. Example of Attributes and Protection and Risk Factors Considered for the Assessment of the Fall Risk

\begin{tabular}{|c|c|c|}
\hline Work Accident & Protection Factors & Attributes \\
\hline \multirow{7}{*}{ Fall risk } & Collective Protection & Safety barriers \\
\hline & Individual Protection & $\begin{array}{l}\text { Harness } \\
\text { Lifeline }\end{array}$ \\
\hline & Risk Factors & Attributes \\
\hline & Work Activity & $\begin{array}{l}\text { Working height } \\
\text { Type of floor } \\
\text { Manual materials handling } \\
\text { Use of tools } \\
\text { Handling of suspended loads }\end{array}$ \\
\hline & Environmental Factors & $\begin{array}{l}\text { Cold } \\
\text { Wind } \\
\text { Rain } \\
\text { Noise } \\
\text { Vibration } \\
\text { Illumination } \\
\text { Dust }\end{array}$ \\
\hline & Psychosocial & $\begin{array}{l}\text { Work pace } \\
\text { Extra Work } \\
\text { Stress }\end{array}$ \\
\hline & Individual & $\begin{array}{l}\text { Auditory insufficiency } \\
\text { Visual insufficiency } \\
\text { Alcohol consumption }\end{array}$ \\
\hline
\end{tabular}

The inference process used for evaluating the risk degree adopts an approach defined in a Fuzzy Multiple Attribute Decision Making model, which stands in an inference chain based on fuzzy rules. The degree of risk for accident is considered to be high if protection measures are missing or 


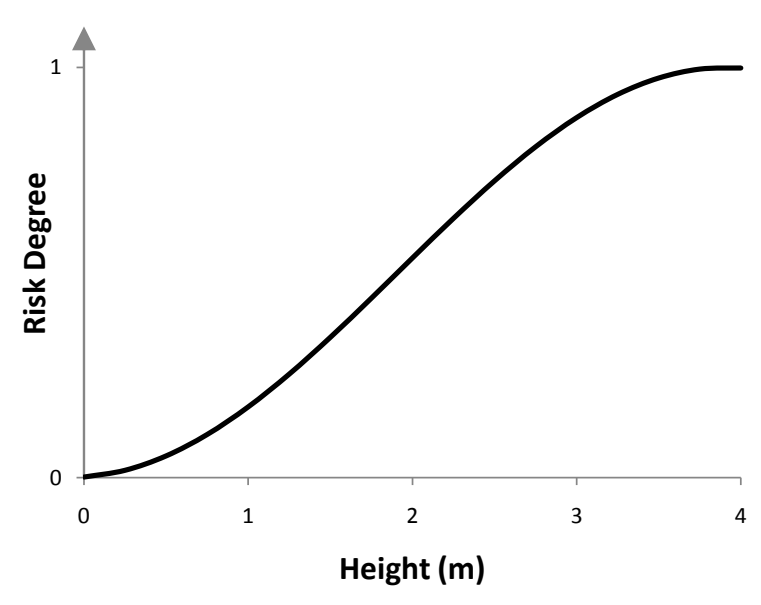

(a)

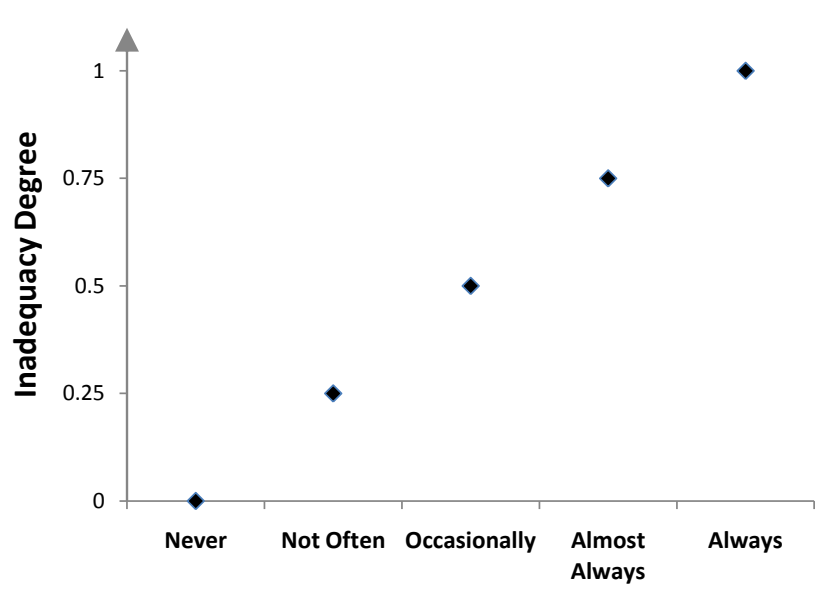

(b)

Fig. (5). (a) Fuzzy set for evaluation of the "working height" attribute; (b) periodicity linguistic variable.

not adequate and simultaneously there are risk factors present on the workplace. The following rule illustrates the assessment of the "fall risk", presented on Table 2.

$$
\begin{aligned}
& \text { IF fall protection is inadequate AND } \\
& \text { fall risk factors are high }
\end{aligned}
$$

THEN fall risk is high

The inadequacy of the protection measures and the severity of the risk factors are evaluated based on relevant attributes. The following rule illustrates the evaluation of the "fall protection inadequacy" for the assessment of "fall risk".

\section{IF safety barrier are inadequate $\mathbf{O R}$ harness use is inadequate $\mathbf{O R}$ lifeline use is inadequate \\ THEN fall protection is inadequate}

The attributes may be evaluated based on objective and/or subjective data. For instance, on the "fall risk" example, the working height is a key factor for the existence of risk. Since the working height is an attribute that can be quantified it is possible to define a fuzzy set its quantification. Fig. (5a) presents an example of fuzzy set to use in such evaluation. On other hand the attributes (including the ones that can be evaluated objectively) can be evaluated based on subjective data using Linguistic Variables. In order to ensure numerical efficiency, in the evaluation process, the linguistic variables terms adopted on AR_X model are discrete fuzzy sets.

Let us consider, for instance, the periodicity Linguistic Variable used to collect the opinions about attributes related for instance with "tools usage" or "alcoholic beverage consumption" frequency. This Linguistic Variable comprehends five linguistic terms that corresponds to risk degrees uniformly distributed over the $[0,1]$ interval. The corresponding discrete fuzzy set (see Fig. 5b) is defined as:

periodicity $=\{0 /$ Never $+0.25 /$ Not often $+0.5 /$ Occasionally +

$$
0.75 / \text { Almost always }+1 / \text { Always }\}
$$

The individual attribute evaluations are aggregated using a logical OR operator. The fuzzy OR operator can however simulate synergistic effect, meaning that the degree of risk resulting from the combination of different risk attributes is higher than any of the individual risk attributes considered.

AR_X results are presented as natural language sentences where the assessed degree of risk is qualified using terms of a linguistic variable, for instance:

The risk of fall is $<q u a l>$

where <qual $>$ is a term (low, medium, high, very high, extreme) from the linguistic variable "risk".

\subsubsection{Fuzzy Inference System}

Guimarães and Lapa developed a fuzzy inference system (FIS) dedicated to risk analysis. The objective is to obtain an understanding of the aging process of a nuclear power system and how it affects the overall plant safety [12].

They developed a ranking Failure Mode and Effects Analysis (FMEA), using a direct method with one expert opinion. They also used a fuzzy inference engine with fuzzy IF-THEN rules to determine a mapping from fuzzy sets in the input universe of discourse to fuzzy sets in the output universe of discourse. The risk priority number was calculated and compared to fuzzy risk priority number using scores from expert opinion to probabilities of occurrence, severity and not detection. This FIS was applied to study the standard four-loop pressurized water reactor containment cooling system. The results demonstrated the potential of the inference system for using the FMEA in aging studies.

\subsubsection{Fuzzy Multi-Attribute Model}

Grassi et al. develop a fuzzy multi-attribute model for risk evaluation in workplaces. The model is based on the evaluation of five different risk factors: injury magnitude, occurrence probability, sensitivity to Personal Protective Equipment non-utilization, undetectability and sensitivity to maintenance non-execution [13]. The first two factors are introduced as the same as in the classical risk model and the last three risk factors represent the most important aspects of 
human behavior and environmental interaction in an industrial field. The relative importance of the risk factors was done through the assignment of weights by the analyst. To do that the analyst uses a weight scale of seven linguistic variables.

The authors proposed six linguistic variables for the evaluation of the risk factors (Negligible, Definitely low, Lower than high, Higher than low, Definitely high and Maximum) that are present in each activity. The methodology uses "fuzzy TOPSIS" to manage the linguistic judgments of experts and to produce the final ranking of activities. The authors stressed that this methodology made possible to obtain a classification more representative of the management feelings, being able to emphasize some tangled aspects, one being, the relation among PPE utilization, injury magnitude, and occurrence probability.

\subsection{Human Reliability Analysis}

\subsubsection{HEROS}

Richei et al. developed HEROS (Human Error Assessment and Optimization System), which is a fuzzy rule-based expert system applied to the analysis of tasks of personnel operating in a nuclear power plant [14]. HEROS is a fuzzy version of the Technique for Human Error Rate Prediction (THERP), which is a probabilistic approach for the evaluation of human actions.

HEROS provides a procedure which can be employed for the qualitative and quantitative evaluation of human error in operating technical systems. The rule-based expert system considers the factors related with the "management", "manmachine interface", and "working environment" as fuzzy sets in order to make possible the evaluation despite the uncertainty. The knowledge base was derived from results of previous ergonomic and psychological studies.

The evaluation of an action is based on pertinent Performance Shaping Factors (PSFs), which are identified after the description of action and its environmental conditions.

For evaluating the influence of the "management" on human performance a linguistic variable Human Error Probability (HEP) was defined which takes into account criteria such as motivation, training, education, decision hierarchy, safety culture, and team composition. This HEP linguistic variable definition resulted from the transformation into possibilities of the existing HEP (probabilities).

For assessing the "working environment" six PSFs are considered, the climatic load, ambient noise, lighting, day time, hazard and stress. Each PSF can be determined based on several sub-criteria like, for instance, temperature, humidity and work energy expenditure, for "climatic load". As previously, the working environment PSFs are defined as linguistic variables.

For assessment of "man-machine interface" is based on a total of 23 PSFs, that characterize the "task profile" and the "ergonomic design". As previously, the working environment PSFs are defined as linguistic variables.

Table 3 synthesizes the criteria used for the assessment of "management", "man-machine interface", and "working environment".
Table 3. HEROS Criteria

\begin{tabular}{|c|l|l|}
\hline HEP & \multicolumn{1}{|c|}{ MMI Areas } & \multicolumn{1}{c|}{ Linguistic Variables } \\
\hline \hline \multirow{4}{*}{ Action } & Management & HEP \\
\cline { 3 - 3 } & \multirow{4}{*}{ Working Environment } & Climatic Load PSF \\
\cline { 3 - 3 } & & Ambient Noise PSF \\
\cline { 3 - 3 } & & Lighting PSF \\
\cline { 3 - 3 } & & Day Time PSF \\
\cline { 3 - 4 } & \multirow{2}{*}{ Man-Machine Interface } & Hazard PSF \\
\cline { 3 - 4 } & & Stress PSF \\
\cline { 3 - 4 } & & Task profile PSF \\
\hline
\end{tabular}

The linguistic variables are used on a set of fuzzy rules that aggregate the data in order to qualitatively evaluate the membership function of the term of the conclusion linguistic variables that characterize the HEP for management, and the PSFs for environment, task profile, ergonomic design and cognitive tasks.

Linguistic variables form the condition of rule which have the general form:

IF (Condition 1 AND ... Condition n) THEN Conclusion m where the logical AND operator is represented by the fuzzy minimum function.

The partial conclusions are then aggregated using a logical OR operator (the fuzzy maximum function). The evaluation of the rules is carried out by a generalized "modus ponens" approximate reasoning process, calculated using the conclusion rules by Mamdani.

The combination of the HEROS with a fault tree analysis allows the identification of the weak points on the manmachine interface and the elaboration of improvement proposals.

\subsubsection{Fuzzy CREAM}

Konstandinidou et al. proposed a fuzzy modeling application for human reliability analysis based on the Cognitive Reliability Error Analysis Method (CREAM) [15]. THERP methodology, referred on the previous example is a first generation method conceived mainly for the nuclear industry. CREAM is an evolution of THERP, which is mentioned as a second generation method based on the cognitive model of human decisions and actions.

CREAM involves an error classification system that integrates individual, technological and organizational factors. This methodology was derived from Contextual Control Model (COCOM), which models the operator performance considering how people are able to maintain control of a situation. COCOM considers four characteristic control modes: scrambled, opportunistic, tactical and strategic.

The control modes provide a fast overall assessment of human reliability, which is combined with a detailed classification of erroneous actions. Such classification 
Table 4. Fuzzy CREAM Parameters

\begin{tabular}{|c|c|c|}
\hline & \multicolumn{2}{|r|}{ Linguistic Variable } \\
\hline & Name (CPCs) & Terms \\
\hline \multirow{9}{*}{ INPUT } & Adequacy of organization & Deficient, Inefficient, Efficient, Very efficient \\
\hline & Working conditions & Incompatible, Compatible, Advantageous \\
\hline & Availability of procedures and plans & Inappropriate, Acceptable, Appropriate \\
\hline & Adequacy of MMI and operational support & Inappropriate, Tolerable, Adequate, Supportive \\
\hline & Number of simultaneous goals & More than actual capacity, Matching current capacity, Less than current capacity, \\
\hline & Available time & Continuously inadequate, Temporarily inadequate, Adequate \\
\hline & Time of day & Night, Day \\
\hline & Adequacy of training and experience & Inadequate, Adequate with limited experience, Adequate with High experience \\
\hline & Crew collaboration quality & Deficient, Inefficient, Efficient, Very efficient \\
\hline OUTPUT & Action Failure probability & Scrambled, Opportunistic, Tactical, Strategic \\
\hline
\end{tabular}

describes the relations between causes and effects. To define sets of possible error modes and probable error causes, instead of PSFs, CREAM uses Common Performance Conditions (CPCs).

In the fuzzy version of CREAM, proposed by Konstandinidou et al., the CPCs are used as linguistic variables, whose terms were defined as triangular fuzzy sets. Table 4 lists the CPCs linguistic variables used as input variables and the corresponding terms used for the description of each parameter. The table also presents the output variable and refers the respective terms.

Following the logic of CREAM, fuzzy rules were developed based on the above mentioned linguistic variables. An example of a fuzzy rule is:

IF

Adequacy of organization is deficient AND
Working conditions are incompatible AND
Availability of procedures and plans is
inappropriate AND
Adequacy of MMI and operational support is
inappropriate AND
Number of simultaneous goals is more than
actual capacity AND
Available time is continuously inadequate AND
Time of day is night AND
Adequacy of training and experience is
inadequate AND
Crew collaboration quality is deficient

THEN Operator will act in a $S C R A M B L E D$ way

The inference process considers the minimum degree of activation for each rule. The output is the union of the effect of all fuzzy rules. The final output is a crisp number for the human error probability obtained by defuzzifying the output fuzzy set.

This way, the application user supplies the input values for a specific working environment and the system estimates the possibility that the operator will perform an erroneous action within the specific context.

\subsection{Manual Material Handling}

Genaidy et al. developed a study regarding Manual Materials Handling (MMH) activities [16]. This study focused on the classification of load heaviness in terms of workers' perceived load using FST. The study was conducted on male and female workers in the package delivery industry to estimate the amount of load that corresponds to various linguistic terms that express load heaviness (e.g. light, medium, somewhat heavy, heavy, etc.).

Employees were asked to estimate the most representative amount of load that could be assigned to various levels of load heaviness as defined according to the Borg Scale. The distribution of amount of load handled for each heaviness level was modeled using a method based on the distribution of demarcation points between levels, which allowed for the calculation of fuzzy set membership functions.

The calculation process involved the computation of means and standard deviations for the various load heaviness levels, and for the demarcation points. Intermediate distribution values were then computed for membership functions using a cumulative normal distribution function. The resulting membership functions were normalized scaling their maximum values to 1 .

The findings pointed for the relationship between linguist terms and amount of load handled, considering a 1.0 certainty factor for the combined membership functions (male and female), presented on Table 5.

The determination of distribution functions for the linguistic terms of ergonomic risk factors is relevant since it can assist researchers and practitioners in establishing rulebased systems for MMH risk assessment.

\subsection{Work-related Musculoskeletal Disorders}

\subsubsection{Fuzzy Linear Regression Model}

McCauley-Bell et al. presents a study for the development of a Fuzzy Linear Regression (FLR) model to predict the relationship of known risk factors to the onset of 
upper body Work-Related Musculoskeletal Disorders (WRMD) [17].

According to the authors the use of FLR analysis presents some advantages, namely:

- Is based on the possibility theory that describes the ambiguity of events or the degree to which they occur;

- $\quad$ Is effective for small data sets;

- It can accommodate the inaccuracy and distortion introduced by linearization. The linear regression model itself contains vagueness, which is interpreted as a regression band instead of a regression line.

The developed model focused on WRMD in office environment, and identified a total of 30 primary risk associated with task, joint deviation, personal, and anthropometric risk factors categories. Some were later on discarded. The final risk factor list is presented in Table 6. The same table presents the relative weights $\left(w_{i}\right)$ that were determined for each risk factor category using an AHP
Table 5. Study Results Relating Linguist Terms and Amount of Load Handled

\begin{tabular}{|c|c|}
\hline Linguistic Term & Load Handled $[\mathbf{k g}]$ \\
\hline \hline Negligible & 0 \\
\hline Very very light & 2.5 \\
\hline Very light & 4.5 \\
\hline Light & 7.5 \\
\hline Moderate & 14 \\
\hline Somewhat heavy & 23 \\
\hline Heavy & 32 \\
\hline Very heavy & 43 \\
\hline Very very heavy & 56 \\
\hline Maximum & 110 \\
\hline
\end{tabular}

analysis, after consultation of 3 ergonomic experts. The result of the linear model can be represented as

Table 6. Identified Primary Risk Factors Per Category

\begin{tabular}{|c|c|c|c|c|}
\hline Risk Factor Category & Relative Weight & & Risk Factor & \\
\hline Task & $w_{l}=0.497$ & $\begin{array}{l}x_{1}: \\
x_{2}: \\
x_{3}: \\
x_{4}: \\
x_{5}: \\
x_{6}: \\
x_{7}: \\
x_{8}: \\
x_{9}:\end{array}$ & $\begin{array}{c}\text { Total typing hours (per day) } \\
\text { Typing speed } \\
\text { Continuous typing time } \\
\text { Hand temperature } \\
\text { Comfortability of workstation orientation and height } \\
\text { Work surface hardness } \\
\text { Hand rest time } \\
\text { Rating of perceived exertion } \\
\text { Working under pressure }\end{array}$ & $\begin{array}{c}(0.231,0) \\
(0.144,0) \\
(-0.078,0) \\
(0.039,0.298) \\
(-0.133,0.044) \\
(-0.152,0.115) \\
(0.059,0) \\
(-0.007,0) \\
(-0.001,0) \\
(0.051,0)\end{array}$ \\
\hline Joint deviation & $w_{2}=0.319$ & $\begin{array}{l}x_{1}: \\
x_{2}: \\
x_{3}: \\
x_{4}: \\
x_{5}:\end{array}$ & $\begin{array}{c}\text { Wrist ulnar deviation } \\
\text { Wrist flexion } \\
\text { Wrist extension } \\
\text { Forearm flexion } \\
\text { Hand suspension }\end{array}$ & $\begin{array}{c}(0.259,0.105) \\
(0.095,0.136) \\
(0.125,0.25) \\
(0.086,0.232) \\
(0.036,0) \\
(-0.005,0)\end{array}$ \\
\hline Personal & $w_{3}=0.110$ & $\begin{array}{l}x_{1}: \\
x_{2}: \\
x_{3}: \\
x_{4}: \\
x_{5}: \\
x_{6}:\end{array}$ & $\begin{array}{c}\text { Age } \\
\text { Medical history } \\
\text { Alcohol usage } \\
\text { Smoking } \\
\text { Years on the job } \\
\text { Previous trauma disorders }\end{array}$ & $\begin{array}{c}(0.198,0.121) \\
(-0.032,0) \\
(0.012,0) \\
(-0.024,0) \\
(0.033,0) \\
(0.042,0) \\
(0.17,0.352)\end{array}$ \\
\hline Anthropometric & $w_{4}=0.073$ & $\begin{array}{l}x_{1}: \\
x_{2}: \\
x_{3}: \\
x_{4}: \\
x_{5}: \\
x_{6}: \\
x_{7}:\end{array}$ & $\begin{array}{c}\text { Body mass index } \\
\text { Hand length } \\
\text { Hand breath } \\
\text { Hand thickness } \\
\text { Wrist circumference } \\
\text { Joint circumference } \\
\text { Maximum pinch strength }\end{array}$ & $\begin{array}{c}(0.283,0.675) \\
(0.046,0.11) \\
(-0.102,0) \\
(0.101,0) \\
(-0.119,0) \\
(0.087,0) \\
(-0.011,0) \\
(-0.144,0)\end{array}$ \\
\hline
\end{tabular}


$Y=w_{1} y_{1}+w_{2} y_{2}+w_{3} y_{3}+w_{4} y_{4}$

where $y_{1}, y_{2}, y_{3}$, and $y_{4}$ are, respectively, the results of the fuzzy linear regression for task-, joint deviation -, personal-, and anthropometric-related risk factors.

Furthermore Table 6 presents the fuzzy parameters for a linear regression level at a confidence (or threshold) level $\mathrm{h}=0.5$ for each of the risk factors. The fuzzy parameters are represented as an ordered pair $A_{i}=\left(\alpha_{i}, c_{i}\right)$, where the $\alpha_{i}$ parameter denotes the center value for the regression coefficient and the $c_{i}$ parameter is the width or fuzziness of the $i^{\text {th }}$ parameter. The first pair of values of each category represents the $A_{0}$ ordered pair of a fuzzy linear regression formulated as follows:

$y=A_{0}+A_{1} x_{1}+A_{2} x_{2}+\ldots+A_{n} x_{n}$

In fuzzy linear regression analysis, the outputs are still fuzzy numbers with a center value and a width. For the defuzzification process the center value it will be adopted.

The results of the study were validated on a small validation group.

\subsubsection{ERGO $X$}

Nunes developed the ERGO_X, which is a modular fuzzy expert system intended to support ergonomic workstation analysis, namely regarding Posture and WRMD $[9,18]$.

The assessment of the ergonomic inadequate situations is based on the assessment of the risk factors, which are evaluated using attributes. Table 7 shows the Attributes and Physical Risk Factors considered for the evaluation of the Carpal Tunnel Syndrome which is an example of WRMD.

Table 7. Example of Attributes and Risk Factors considered for evaluation of Physical Risk Factors of the Carpal Tunnel Syndrome

\begin{tabular}{|c|c|c|}
\hline WRMD & Physical Risk Factor & Attribute \\
\hline \multirow{10}{*}{$\begin{array}{c}\text { Carpal } \\
\text { Tunnel } \\
\text { Syndrome }\end{array}$} & \multirow{3}{*}{ Posture } & Wrist flexion/extension \\
\hline & & Wrist ulnar/cubital deviation \\
\hline & & Hand pressure type /grip-keying \\
\hline & Force & Wrist/hand force \\
\hline & \multirow{2}{*}{ Repetition } & Wrist/hand frequent movements \\
\hline & & Finger flexion frequent movements \\
\hline & Cold & Wrist/hand cold \\
\hline & Vibration & Hand/arm vibration \\
\hline & \multirow{2}{*}{ Contact Pressure } & Wrist contact pressure type \\
\hline & & Hand palm contact pressure type \\
\hline
\end{tabular}

ERGO_X assessment is performed based on an inference process involving a chain of production rules, like the one that is presented to illustrate the evaluation of the "Carpal Tunnel Syndrome".

IF Wrist flexion/extension is inadequate OR Wrist ulnar/cubital deviation is inadequate
OR Hand pressure type /grip-keying is

inadequate

THEN posture is inadequate

$\cdots$

IF Hand/arm vibration is inadequate

THEN vibration is inadequate

IF posture is inadequate $\mathbf{O R}$... OR vibration is inadequate

THEN there is a possibility for occurrence of Carpal Tunnel Syndrome

The OR operators are fuzzy union operators, meaning that condition truth degrees are aggregated producing a new truth degree on the interval $[0,1]$. Since truth degrees are quantifiable the logical operators can be defined as mathematical expressions and the results computed numerically. For instance, the ERGO_X uses as OR operator the Dubois and Prade Union $\left(\mu_{A \alpha^{\prime} B}\right)$ defined on the following expression:

$$
\mu_{A \alpha^{\prime} B}=\frac{\mu_{A}+\mu_{B}-\mu_{A} \mu_{B}-\min \left(1-\alpha^{\prime}, \mu_{A}, \mu_{B}\right)}{\max \left(1-\mu_{A}, 1-\mu_{B}, \alpha^{\prime}\right)}, \alpha^{\prime} \in[0,1]
$$

where $\mu_{A}$ and $\mu_{B}$ are the truth degrees (in this context referred as inadequacy degrees) to aggregate using the operator. Since this is a parametric operator we can simulate specific behaviors, like synergy, tuning the value $\alpha^{\prime}$.

ERGO_X results are presented as natural language sentences where the assessed degree of risk is qualified using terms of a linguistic variable, for instance:

The possibility for occurrence of Carpal Tunnel Syndrome is $<$ qual $>$

where <qual> is a term (low, medium, high, very high, extreme) from the linguistic variable "risk".

ERGO_X system has been applied on the Ergonomic analysis of workstations, mainly on office and industry environment. The validation process of the knowledge base was performed by comparison of ERGO_X results against the results produced by other evaluation methods, and against data from occupational physicians [19]. Later a software based on this model, FAST ERGO_X was developed [20].

\subsection{Layout Design}

Hanson et al. propose a fuzzy comfort model to integrate in human simulation tools in order to reduce the need for ergonomic tests in real physical environments [21]. The application context is the design of a car interior, namely regarding the location of controls. The motivation for this work was the awareness that the car interior is a complex man-machine interface, and that poorly designed interiors contribute to traffic accidents as well as discomfort and disorders in professional and long distance drivers.

The authors used a fuzzy clustering process to validate the assumption that the perceived comfort is related with physical parameters from the human anthropometry (the 
body stature) and the technical system, i.e. the control position (related with the reach distance). The study started with 744 samples of perceived comfort. The outliers were identified and removed from the data set using the least trimmed squares regression resulting on a data pool of 652 samples. These samples were randomly separated on two sets of equal size. The first set was the training set used to perform the clustering and to estimate the model parameters. The second set was applied to check the performance of the achieved model. The model accuracy estimators were the average, the standard deviation and the root mean square error. Four variables were used to model the input-output relation: the height $(\mathrm{x})$ and side $(\mathrm{z})$ coordinates of the control position, and the body stature and shoulder-finger tip length.

Each sample fuzzy membership value was calculated using a distance measure to the cluster centers. The clustering identified three comfort levels, very comfortable, neutral and very discomfortable.

Common perceived comfort estimation models used on human CAD tools are based on body posture and joint inclination. As mentioned, the fuzzy model uses individual anthropometric data and control position as inputs, which when combined are similar to the body posture. Nevertheless, as Hanson et al. highlighted, driver's anthropometric data and control positions are easier to access than body postures and angles between segments. This way, the model opens room for the development of ergonomic evaluation tools combined with human simulation tools. Such tools lead to minimize development costs, due to fewer resources allocated for experimental evaluation, e.g. the use of mock-ups.

Using this type of models, the ergonomic problems can be identified early in the design process, avoiding the resulting high costs if discovered late in the design process.

\section{CONCLUSIONS}

This paper is based on a literature review of the application of FL in HCS from 1998 to 2009, using a keyword search in several databases.

Ten examples of application of Fuzzy Logics approaches to the assessment of Human-Centered Systems were presented, four being related with Risk Assessment for Work Accidents, two with Human Reliability Analysis, one with Manual Material Handling, two with Work-related Musculoskeletal Disorders and one with Layout design. Most systems adopted a rule-based or a multiple attribute methodology to address the evaluation problem, but other systems followed different methods such as fuzzy linear regressions or fuzzy clustering processes.

Despite the differences on the methodologies adopted and on the fields of application all authors stress the benefits resulting from the use of Fuzzy Logics. There is unanimity about the usefulness of using Fuzzy Logics in addressing the uncertainty associated with the definition and modeling of HCS. This is particularly important since ergonomic and safety risk analysis raise decision making problems of illdefined nature, due to the complexity of the intervening factors, the context dependencies, or the subjectivity of some of the processed data, generally collected using linguistic information.
Fuzzy Sets and Fuzzy Logics is a generalization of Classical Sets and Boolean Logics that extends their potential providing a mathematical framework for the systematic treatment of vagueness and imprecision. In general terms a Fuzzy approach can do whatever is a Classical approach does and can go beyond. Fuzzy Logics facilitates the elicitation and encoding of uncertainty-related knowledge; offers a flexible representation mechanism for dealing with vague data that allows a seamless and coherent integration of subjective and objective data; eliminates the problem of using interval categories allowing the use of continuous ranges of values; and by provides a host of aggregation operators that can simulate several behaviors, such as synergy. Maybe this is the reason why no references were found refuting the merits of a Fuzzy Logics approach.

This paper is a small sample of the attention that Fuzzy Systems is attracting both from academics and software developers to deal with complex issues from a great variety of knowledge domains. Some methodologies (for example, fuzzy rule-based systems and fuzzy expert systems) are commonly used, but the flexibility that Fuzzy Logics offers allows the adoption of much diversified methodologies according to authors' expertise and the problem domains on hand. This feature allows an easier approach in addressing quite complex tasks such as knowledge acquisition, knowledge representation, and decision-making modeling required for developing human-centered systems.

The importance of improving HCS is critical due the personal, social and financial impacts (illness, injuries, material damage, productivity loss and liabilities) on society and on companies due to accidents, disorders and errors resulting of poorly designed work environments, systems and procedures.

\section{REFERENCES}

[1] Karwowski W, Mital A. Fuzzy concepts in Human Factors/Ergonomics research. In: Karwowski W, Mital A, editors. Applications of Fuzzy Set Theory in Human Factors. Amsterdam: Elsevier Science Publishers B.V. 1986; pp. 41-54.

[2] Zadeh LA. Fuzzy Logic = Computing with words. IEEE Transactions on Fuzzy Systems. 1996; 4(2): 103-11.

[3] Zadeh LA. Fuzzy sets. Inform Control 1965; 8: 338-53.

[4] Nunes IL. Fuzzy Multicriteria Model for Ergonomic workplace analysis and Risk analysis. Information Technology, Knowledge Management and Engineering for Enterprise Productivity and Quality of Working Life (International Conference: ComputerAided Ergonomics and Safety - CAES'05) 2005 25-28; Mai, Kosice-Slovak Republic.

[5] Zadeh LA. The concept of a linguistic variable and its application to approximate reasoning-part I. Inf Sci 1975; 8: 199-249.

[6] Zadeh LA. PRUF-a meaning representation language for natural languages. Int J Man Mach Stud 1978; 10: 395-460.

[7] Zadeh LA. Linguistic variables, approximate reasoning and dispositions. Med Inform 1983; 8(3): 173-86.

[8] Zadeh LA. Test-score semantics as a basis for a computacional approach to the representation of meaning. In: Yager RR, Ovchimnikov S, Tong RM, Nguyen HT, Eds. Fuzzy Sets and Applications: Selected Papers by L A Zadeh: John Wiley and Sons 1987; pp. 655-83.

[9] Nunes IL. ERGO_X - The Model of a Fuzzy Expert System for Workstation Ergonomic Analysis. In: Karwowski W, Ed. International Encyclopedia of Ergonomics and Human Factors: CRC Press 2006; pp. 3114-21.

[10] Zimmermann H-J. Fuzzy Set Theory and Its Applications. $3^{\text {rd }}$ ed. Kluwer Academic Publishers; 1996.

[11] Gentile M, Rogers W, Mannan M. Development of an Inherent safety Index Based on Fuzzy Logic. AIChE J 2003; 49(4): 959-68. 
[12] Guimarães ACF, Lapa CMF. Fuzzy inference to risk assessment on nuclear engineering systems. Appl Soft Comput 2007; 7: 17-28.

[13] Grassi A, Gamberini R, Mora C, Rimini B. A fuzzy multi-attribute model for risk evaluation in workplaces. Saf Sci 2009; 47: 707-16.

[14] Richei A, Hauptmanns U, Unger H. The human error rate assessment and optimizing system HEROS - a new procedure for evaluating and optimizing the man-machine interface in PSA. Reliability Engin Sys Saf 2001; 72: 153-64.

[15] Konstandinidou M, Nivolianitou Z, Kiranoudis C, Markatos N. A fuzzy modeling application of CREAM methodology for human reliability analysis. Reliability Engin Sys Saf 2006; 91: 706-16.

[16] Genaidy AM, Karwowski W, Christensen D, Vogiatzis C, Deraiseh N, Prins A. What is "heavy"? Ergonomics 1998; 41(4): 420-32.

[17] McCauley-Bell P, Crumpton LL, Wang H. Measurement of cumulative trauma disorder risk in clerical tasks using fuzzy linear regression. IEEE Trans Syst Man Cybern C Appl Rev 1999; 29(1): $1-14$.

[18] Nunes IL. National Patent $\mathrm{n}^{\circ}$ 103446: Método de Análise Ergonómica de Postos de Trabalho. [Workstation Ergonomic Analysis Method]; 2009.

[19] Nunes IL. Modelo de Sistema Pericial Difuso para Apoio à Análise Ergonómica de Postos de Trabalho [Fuzzy Expert System Model to Support Workstation Ergonomic Analysis] [PhD Dissertation]. Lisbon, Portugal: Universidade Nova de Lisboa 2003.

[20] Nunes IL. FAST ERGO_X - a tool for ergonomic auditing and work-related musculoskeletal disorders prevention. WORK: J PrevAssessm Rehabil 2009; 34(2): 133-48.

[21] Hanson L, Willfried W, Sperling L. A control handling comfort model based on fuzzy logics. Int J Ind Ergon 2003; 31: 87-100.

(C) Isabel L. Nunes; Licensee Bentham Open.

This is an open access article licensed under the terms of the Creative Commons Attribution Non-Commercial License (http: //creativecommons.org/licenses/ by-nc/3.0/) which permits unrestricted, non-commercial use, distribution and reproduction in any medium, provided the work is properly cited. 